\title{
GENERIC DIRICHIET POLYGONS AND THE MODULAR GROUP
}

\author{
by A. M. MACBEATH
}

Dedicated to Robert Rankin, teacher, colleague and friend on his seventieth birthday

1. Introduction. The concept of "marked polygon", made explicit in this paper, is implicit in all studies of the relationships between the edges and vertices of a fundamental polygon for Fuchsian group, as well as in the topology of surfaces. Once the matching of the edges under the action of the group is known, one can deduce purely combinatorially the distribution of the vertices into equivalence classes, or cycles. Knowing a little more, the order of the rotation group fixing a vertex in each cycle, we can write down a presentation for the group.

It was recognized by Poincaré that, when we have a polygon which is not a priori associated with a group, but when a matching of its edges is known and certain simple metric conditions, one of which he overlooked, are satisfied, then a group with the given polygon as fundamental polygon can be constructed. This is the celebrated Poincare Existence Theorem, for which a complete satisfactory proof has been published only as recently as 1971, independently by Maskit and de Rham ([6], [12]).

If we choose a fixed base-point in the hyperbolic plane, then for every discrete isometry group $\Gamma$ we can form the Dirichlet polygon by selecting only those points of the plane which are at minimal distance, among points in the same $\Gamma$-orbit, from $p$. We then have an edge-matching for this polygon. The distribution of groups in a given isomorphism class between the various combinatorially possible edge-matchings is of interest in itself and will probably help us to understand related questions such as the action of the Mapping Class Group on Teichmüller space.

The first obvious question to be answered is which combinatorially possible edgematchings can actually be realized by Dirichlet polygons. In $\S 8$ we answer this question for groups with a fundamental region of finite area with a Realization Theorem which asserts that essentially all markings are possible. We use the theorem of Beardon on the existence of hyperbolic polygons with prescribed angles and having an inscribed circle. Beardon's polygon almost miraculously has precisely the properties we need to show that it is a Dirichlet polygon.

In the last part of the paper we specialize to study fundamental groups of compact orientable surfaces, associated with algebraic curves. Almost all Dirichlet polygons in this case have a generic matching, that is, all vertex cycles have length 3 . The technique used to prove the Realization Theorem picks out, for each generic matching, a specially symmetric Dirichlet polygon, defining what we call a central curve for each edgematching. We prove that all central curves can be uniformized by subgroups of the modular group.

2. Notation and terminology. For the mere statement of the Realization Theorem to make sense it is essential that we should define separate entities, combinatorial and 
geometric. The importance of this in the context of the Existence Theorem was clearly recognised by Poincare ([14], p. 127). In the context of the modular group, Millington [13] recognised that edge-matchings are essentially pairs of permutations. More recently, Hussein ([8]) made a detailed study of generic matchings in genus 2 and 3, regarding them simply as pairs of elements of $S_{n}, n$ being the number of vertices of the polygon. To include orientation-reversing isometries, we here use permutations of degree $2 n$, acting on the set of directed edges. The reduction to degree $n$ in the orientation-preserving case is made in $\$ 9$.

We distinguish therefore between the edge-matching, the topological marked polygon and the geometric marked polygon. We also distinguish two concepts of "polygon"topological and geometric. The geometric polygons are always convex, for that is all we need, and only their extreme points are counted as vertices.

We use the term "Dirichlet polygon" for the closure of the Dirichlet region rather than as a synonym. The closure, after all, carries all the information about the group. The term weight is used for the order of the orientable stabilizer of a vertex, rather than the word "order", which is over-used in mathematics and therefore ambiguous. Otherwise I have adhered to the terminology of [2], [9], with Magnus's notation [11] for triangle groups and Coxeter's [4], [5] for tessellations.

3. Polygons. A polygon $P$ is a disc $B^{2}$ together with a finite subset $V \subset \delta B^{2}$. The points of $V$ are the vertices of $P$, and, if there are $n$ of them, $P$ is an $n$-gon. We shall always assume that $n \geqslant 3$. An edge of $P$ is the closure of a component of $\delta B^{2} \backslash V$ and a directed edge is a pair $(e, v)$ where $e$ is an edge and $v$ is one of the two vertices in $e$. We call $v$ the initial point or initial vertex of the directed edge. Let $E(P)$ denote the set of $2 n$ directed edges. The set of $n$ undirected edges, when we need to refer to it, is denoted by $E_{0}(P)$. If $(e, v)$ is a directed edge, there is precisely one edge $e^{\prime}$, say, other than $e$, which has $v$ as a vertex, and precisely one vertex $v^{\prime}$, say, other than $v$, of the edge $e$. Define permutations

$$
\rho, \nu: E(P) \rightarrow E(P) \quad \text { by } \quad \rho(e, v)=\left(e, v^{\prime}\right), \quad \nu(e, v)=\left(e^{\prime}, v\right) .
$$

The function $\rho$ is direction Reversal, $\nu(e, v)$ is a Neighbouring directed edge. Both $\rho$ and $\nu$ are regular permutations of order 2. Their composite $\sigma=\nu \rho$ ( $\rho$ first, then $\nu$ ) is a regular permutation of order $n, \sigma(e, v)$ being the Successor of $(e, v)$ in the cyclic order of directed edges indicated by the choice of $v$ as initial point.

The set $E(P)$, together with the pair of permutations $\rho, \nu$ is a convenient algebraic model for the polygon. The undirected edges can be identified with elements of the quotient set $E(P) /\langle\rho\rangle$, the set of vertices with $E(P) /\langle\nu\rangle$. Working mainly with directed edges, we shall use the single Greek letter $\eta$, with or without suffixes, for a directed edge. Then $|\eta|$ will denote the corresponding undirected edge and $i(\eta)$ will denote its initial point. Thus $\eta=(|\eta|, i(\eta))$.

In terms of vertices, if $v$ and $v^{\prime}$ are the two vertices of the same undirected edge $e$, then $\left(v, v^{\prime}\right)$ will denote the directed edge $(e, v)$. Thus $\left(v^{\prime}, v\right)=\rho\left(v, v^{\prime}\right)$. 
An isomorphism of one $n$-gon $(P, V)$ with another $\left(P^{\prime}, V^{\prime}\right)$ is a homeomorphism of the topological pair $(P, V)$ onto $\left(P^{\prime}, V^{\prime}\right)$, an automorphism being as usual a selfisomorphism. Each automorphism induces a permutation of $E(P)$ which commutes with both $\rho$ and $\nu$. The group $\operatorname{Aut}(E(P))$ of permutations which commute with both $\rho$ and $\nu$ is called the automorphism group of the set of directed edges. It is the quotient of the automorphism group of the polygon modulo those which are isotopic to the identity and can essentially be regarded as the automorphism group of $P$.

Since $\langle\rho, \nu\rangle$ acts regularly and transitively on $E(P)$, its action on $E(P)$ is equivalent to the Cayley representation of the dihedral group on itself by left translation. In this model, Aut $(E(P))$ is the group of right translations. However, $\rho$ and $\nu$ are not automorphisms of $E(P)$ and there is no canonical isomorphism between $\langle\rho, \nu\rangle$ and the automorphism group.

4. Marked polygons, cycles. Let $P$ be a polygon and let $\mu_{0}$ be a permutation of $E_{0}(P)$ such that $\mu_{0}^{2}=$ id. Suppose that, to each $e \in E_{0}(P)$ there is assigned a homeomorphism $T(e): e \rightarrow \mu_{0}(e)$ such that

$$
T\left(\mu_{0}(e)\right)=T(e)^{-1} .
$$

The collection $\left(P, \mu_{0},\left\{T(e): e \in E_{0}(P)\right\}\right)$ is called a topologically marked polygon and $\left(\mu_{0},\{T(e)\}\right)$ is a topological marking of $P$. The homeomorphisms $T(e)$ are called matching homeomorphisms.

If $v, v^{\prime}$ are the two vertices of an edge $e$, then $T(e)$ maps these to vertices $v_{1}, v_{1}^{\prime}$ of $\mu_{0}(e)$ respectively, thus inducing a permutation $\mu$ of directed edges given by $\mu\left(v, v^{\prime}\right)=$ $\left(v_{1}, v_{1}^{\prime}\right)$. Clearly $\mu \rho=\rho \mu$ and, by $4.1, \mu^{2}=\mathrm{id}$.

DEFINITION 4.2. An edge-matching of $P$ is a permutation $\mu: E(P) \rightarrow E(P)$ such that $\mu^{2}=\mathrm{id}, \mu \rho=\rho \mu$. A marked polygon is a pair $(P, \mu)$ where $P$ is a polygon and $\mu$ an edge-matching.

A topologically marked polygon induces an edge-matching $\mu$. The matching $\mu$ determines the pairing $\mu_{0}$ of undirected edges and we shall often write $(P, \mu,\{T(e)\})$ instead of $\left(P, \mu_{0},\{T(e)\}\right)$. Unlike $\nu, \rho$, neither $\mu_{0}$ nor $\mu$ need be regular permutations. In the classical case of groups generated by reflections, $\mu=\mu_{0}=\mathrm{id}$.

DEFINITION 4.3. The quotient space of the topologically marked polygon is the space $P / q$ where $q$ is the equivalence relation generated by identifying $T(e) x$ with $x$ for each point $x$ on each edge $e$.

A point $x$ in the interior of $P$ belongs to a singleton equivalence class, and a relatively interior point $y$ of an edge $e$ is equivalent to $T(e) y$ and to no other point. Consider next a vertex $v=i(\eta)=i(\nu \eta)$. By definition, $v$ is q-equivalent to the initial points of both $\mu \eta$ and $\mu \nu \eta$. In fact, two directed edges have q-equivalent initial points if and only if they belong to the same orbit under the dihedral $\dagger$ group $\langle\mu, \nu\rangle$. The same vertex, not merely

$\dagger$ The cyclic group $C_{2}$ and of course the 4-group $C_{2}+C_{2}$ are here given honorary membership of the club of dihedral groups. 
q-equivalent vertices, is initial point of $(\mu \nu)^{r} \eta$ and $\nu(\mu \nu)^{r} \eta$. So we do not need the whole dihedral group to generate the q-class of $v$, but only its cyclic subgroup of index 2 generated by $\tau=\mu \nu$.

DEFINITION 4.4. An edge-cycle is a cycle of directed edges under the action of the permutation $\tau$. A vertex-cycle is the set of initial vertices of an edge-cycle.

We obtain the same vertex cycle (as a set-the cyclic order is opposite) regardless of which of the two edges with initial vertex $v$ we choose. If $\boldsymbol{\eta}$ is a directed edge and $v$ is its initial vertex, the number of elements in the $\tau$-cycle of $\eta$ is called the cycle-length of $\eta$ (or $v$ ) and denoted by $l(\eta)$ (or $l(v)$ ). It is not necessarily the number of elements in the vertex cycle of $v$-it may be equal or it may be double that number.

5. Hyperbolic geometry. Let $U, \bar{U}, \delta U$ denote respectively the interior, closure and boundary of the unit disc $U=\{z \in \mathbb{C}:|z|<1\}$. Points of $U$ will be taken as points of the hyperbolic plane, the infinitesimal metric being given by $2|d z| /\left(1-|z|^{2}\right)$ and points of $\delta U$ will be called ideal points. Geodesics in this metric are circles or lines orthogonal to $\delta U$ and will simply be called lines. When we need to talk about euclidean straight lines or circles, we shall use the adjective euclidean. Otherwise all references to circles, lines, half-planes, convex sets and so forth will apply in the sense of hyperbolic geometry. The geodesic hyperbolic distance between two points $p, q$ of $U$ will be denoted by $d(p, q)$. Sometimes we shall refer to points of $U$ as ordinary points, $\ddagger$ to distinguish them from ideal points. $G$ will denote the group of all isometries of $U$ and $G^{+}$the subgroup of $G$ of index 2 which preserves orientation. Elements, other than the identity, of $G^{+}$are of three kinds:

(i) rotations, or elliptic elements with a single fixed point in $U$, no fixed point on $\delta U$,

(ii) parabolic elements, with a single fixed point on $\delta U$, no fixed point in $U$,

(iii) hyperbolic elements, with two fixed points on $\delta U$, no fixed point in $U$. Hence:

5.1. If an element of $G^{+}$has on fixed point in $U$ and another on $\delta U$, then it is the identity.

If $v$ is an ideal point, a $v$-horocycle is a euclidean circle internally tangent to $\delta U$ at $v$. Parabolic elements fixing $v$ map each $v$-horocycle to itself. This property characterizes them in the following strong sense which we shall need later.

Lemma 5.2. Let $v \in \delta U, w \in U, T \in G^{+}, T v=v$. Then $T$ is parabolic if and only if $w$, Tw lie on the same $v$-horocycle.

Proof. If $w$ and $\mathrm{T} w$ lie on the same $v$-horocycle, there is a parabolic transformation $V$, say, which maps $v$ on $v$ and $w$ on $T w$. Then, since $T^{-1} V$ fixes $v \in \delta U, w \in U$, we have $T^{-1} V=$ id, by $5.1, T=V$.

$\ddagger$ This is a slight departure from the usual terminology if one has a group with a proper subset of $\bar{U}$ for its limit set; but we do not consider such groups in this paper. 
LEMMA 5.3. If $L_{1}$ and $L_{2}$ are two lines meeting at the ideal point $v$ and if a circle (euclidean or non-euclidean-it comes to the same) touches $L_{1}, L_{2}$ at $p_{1}, p_{2}$ then $p_{1}, p_{2}$ lie on the same $v$-horocycle.

Proof. The euclidean common tangents at $p_{1}, p_{2}, v$ to the three euclidean circles touching in pairs at these three points are the three radical axes and therefore meet at a point 0 . The desired horocycle is a euclidean circle centre 0 .

6. Dirichlet polygons, weighted marked polygons. Let $\Gamma$ be a discrete subgroup of $G, p$ an ordinary point whose $\Gamma$-stabilizer is trivial. Let $D(\Gamma, p)=\{z \in U: d(z, p)<$ $d(z, \gamma), 1 \neq \gamma \in \Gamma\}$, and let $F(\Gamma, p)$ be the closure in $\bar{U}$ of $D(\Gamma, p)$. Then $D(\Gamma, p), F(\Gamma, p)$ are called the Dirichlet region and the Dirichlet polygon respectively for $\Gamma$ at $p$. The Dirichlet polygon, which, when possible, we denote simply by $F$, is a convex set whose boundary is a union of line-segments and ideal points. In general there could be a countable infinity of bounding line-segments, and an ideal boundary point of the "polygon" need not be the endpoint of a line-segment. However, Siegel [15] proved that if the area of the quotient $U / \Gamma$ is finite, then $F$ is indeed a polygon in our sense-a convex polygon bounded by a finite union of line-segments, some of which may have ideal endpoints. Henceforth we shall restrict attention to this case. The edges of the polygon will be maximal straight segments, and the only points regarded as vertices will be the endpoints of such segments. Those vertices which are ideal points will be called ideal vertices, the others ordinary vertices. If $e$ is an edge of $F$, then there is a unique $\gamma_{e} \in \Gamma$ such that $e=F \cap \gamma_{e} F$. Let $T(e)=\gamma_{e}^{-1}$. Since $\gamma_{e}^{-1} e=F \cap \gamma_{e}^{-1} F, \gamma_{e}^{-1} e$ is also an edge of $F$ and $T\left(\gamma_{e}^{-1} e\right)=\gamma_{e}=T(e)^{-1}$. Thus we have a topological marking as at 4.1 of the Dirichlet polygon. This is called the standard marking of $F$ by $\Gamma$ and the associated edge-matching is the standard edge-matching.

If $\eta$ is a directed edge of $F$ with initial vertex $v$, the angle at $\eta$ (or, the angle at $v$ ) is defined to be the angle between $\eta$ and $\nu \eta$ at $v$, taken positive. It is denoted by $\theta(\eta)$ or $\theta(v)$. The sum of the angles at an ordinary cycle of directed edges is an integral submultiple of $2 \pi$, say

$$
\sum_{j=0}^{1(n)-1} \theta\left(\tau^{i} \eta\right)=\frac{2 \pi}{m(\eta)} .
$$

The integer $m(\eta)$ is the same for all $\eta$ in the cycle, and is called the weight of the cycle. It is the order of the stabilizer of $i(\eta)$ in $\Gamma \cap G^{+}$. We extend the terminology to ideal vertices by putting $m(\eta)=\infty$ if $i(\infty)$ is an ideal point. These properties of the Dirichlet polygon, which indeed it shares with any locally finite convex fundamental polygon, motivate the following definition.

6.2. A weighted marked polygon is a triple $(P, \mu, m)$ consisting of a polygon $P$, an edge-matching $\mu$ and a function $m: E(P) /\langle\nu, \mu\rangle \rightarrow \mathbb{N} \cup\{\infty\}$ from the set of vertex-cycles to the set of positive integers extended by including $\infty$.

The Dirichlet polygon, together with the standard edge-matching and the weighting of the edge-cycles given by the angle-sums, forms a weighted marked polygon, which we 
also denote by $F$. By the Gauss-Bonnet Theorem ([2], p. 153, Theorem 7.15.1), its area is

$$
\mu(F)=\pi(n-2)-\frac{1}{2} \sum_{\eta \in E(F)} \theta(\eta)=\pi\left(n-2-\sum_{\eta \in E(F) / \tau\rangle} m(\eta)^{-1}\right) .
$$

The factor $\frac{1}{2}$ is needed because each angle is counted twice, once for each of the two edges enclosing it.

DEFINITION 6.4. If $(P, \mu, m)$ is a weighted marked polygon, its Euler characteristic is defined by the expression

$$
\chi(P, \mu, m)=\frac{1}{2}\left(2-n+\sum_{\eta \in E(F) /(\tau)} m(\eta)^{-1}\right) .
$$

DEFINTION 6.5. Two weighted marked polygons $(P, \mu, m)$ and $\left(P^{\prime}, \mu^{\prime}, m^{\prime}\right)$ are isomorphic if there is a bijection $\varphi: E(P) \rightarrow E\left(P^{\prime}\right)$ such that $\varphi \mu=\mu^{\prime} \varphi, \varphi \nu=\nu \varphi, \varphi \rho=\rho \varphi$ and $m^{\prime} \varphi=m$.

7. Geometric polygons. We now refine the idea of a topological marked polygon by insisting that $P$ should be a convex polygon in $\bar{U}$ and that the matching homeomorphisms should be restrictions of isometries of $U$. The edges of $P$ must be line-segments whose endpoints, perhaps including some ideal points, are the vertices. We adopt the convention that no points of $P$ except vertices are ideal points and that each ordinary vertex is a genuine "corner", not a relatively interior point of any line-segment contained in $\delta P$. The term convex polygon will be used only in this sense.

Suppose then that $P$ is a convex polygon, $\mu_{0}$ a permutation of $E_{0}(P)$ such that $\mu_{0}^{2}=$ id. Suppose that, to each $e \in E_{0}(P)$ is assigned an isometry $T(e)$ of $u$, the assignment to satisfy the following conditions:

$$
\begin{aligned}
& T(e) \text { maps e onto } \mu_{0}(e), \\
& T(e) P \cap P=\mu_{0}(e) \\
& T\left(\mu_{0}(e)\right)=T(e)^{-1} .
\end{aligned}
$$

The transformations $T(e)$ are called matching isometries. By restricting the matching isometries each to its assigned edge $e$, we obtain a topological marking inducing a matching $\mu$ of directed edges. Let $m$ be a weighting of the marked polygon $(P, \mu)$. The entire collection $(P, \mu, m ;\{T(e)\})$ is called a geometric weighted marked polygon if, in addition to 7.1.1, 2, 3, the following two conditions hold:

(7.1.4) If $i(\eta)$ is an ordinary vertex and $l(\eta)=l$, then

$$
\sum_{j=0}^{l-1} \theta\left(\tau^{j} \eta\right)=\frac{2 \pi}{m(\eta)}
$$

(7.1.5) If $i(\eta)$ is an ideal vertex and $l(\eta)=l$, then the isometry $T(|\eta|) T(|\tau \eta|) \ldots T\left(\left|\tau^{1-1} \eta\right|\right)$ is parabolic.

The raison d'être of this elephantine concept is the following notorious theorem. 
7.2. PoINCARÉ EXISTENCE THEOREM. The isometry group generated by the matching isometries of a geometric weighted marked polygon is discrete, and the interior of the polygon is a fundamental region for the group.

For proofs see [6], [12]. De Rham does not formally consider orientation-reversing isometries, but the necessary modifications are immediate.

8. The Realization Theorem. In this section we prove the following result.

8.1. Realzation TheORem. Let $(P, \mu, m)$ be a weighted marked polygon. Then there exists a discrete isometry group $\Gamma$ of $U$ and a point $p \in U$ such that $F(\Gamma, p)$, with the standard weighting and marking, is isomorphic to $(P, \mu, m)$ if and only if

$$
\begin{aligned}
& \chi(P, \mu, m)<0 \\
& l(\eta) m(\eta) \geqslant 3 \text { for all } \eta \in E(P) .
\end{aligned}
$$

Condition 8.1 .1 is necessary by 6.3 . Condition 8.1 .2 is forced on us by our unyielding attitude that only extreme points of the polygon can be called vertices. It could be circumvented at a slight cost, but we prefer not to do so.

Suppose then that $(P, \mu, m)$ is a weighted marked $n$-gon of negative Euler characteristic, and with the property that, for every directed edge $\eta$ we have $l(\eta) m(\eta) \geqslant 3$. Number its vertices, in one of the two possible cyclic orders, $1,2, \ldots, n$. Let $m_{1}, \ldots, m_{n}$ be the weights of these vertices, so that $m_{r}=m_{s}$ if $r$ and $s$ belong to the same vertex-cycle. Let $l_{1}, \ldots, l_{n}$ be the lengths of the edge-cycles defined by edges with initial vertices $1, \ldots, n$, so that again $l_{r}=l_{s}$ if $r$ and $s$ belong to the same cycle. For $j=1, \ldots, n$, set $\alpha_{i}=2 \pi / l_{i} m_{i}$. The condition of negative Euler characteristic can be written in the form

$$
\alpha_{1}+\ldots+\alpha_{n}<(n-2) \pi .
$$

This is precisely the condition needed for the existence of a convex $n$-gon $P_{1}$ in $\bar{U}$ with angles $\alpha_{1}, \ldots, \alpha_{n}$ at vertices $v_{1}, \ldots, v_{n}$ in cyclic order, where $P_{1}$ has an inscribed circle of radius $r$ given by

$$
\sum_{i=1}^{n} \sin ^{-1}\left(\frac{\cos \frac{1}{2} \alpha_{i}}{\cosh r}\right)=\pi
$$

For the elegant proof of this see [1], or [2], p. 155-6. Though Beardon does not say so explicitly, it is implicit in his argument that, if the polygon is to have an inscribed circle, then its radius is uniquely determined by 8.2 and that the polygon is unique up to an isometry.

Let $P_{1}$ be such a polygon. Let $\mu_{1}$ be the edge-matching of $P_{1}$ so chosen that $j \mapsto v_{j}$ is an isomorphism of marked polygons, that is, we must have $\mu_{1}\left(v_{i}, v_{j}\right)=\left(v_{k}, v_{l}\right)$ whenever $\mu(i, j)=\mu(k, l)$, where, of course $|i-j|=|k-l|=1$. For each directed edge $\eta=\left(v_{i}, v_{j}\right)$, let $p(\eta)$ be the point of contact of $|\eta|$ with the inscribed circle, at the foot of the perpendicular from the centre $c$ of the inscribed circle to $|\eta|$. The right-angled triangle with vertices $v_{i}, p(\eta), c$ has a side of length $r$ opposite to $v_{i}$ and angle $\frac{1}{2} \alpha_{i}$ at $v_{i}$, so the 
distance $d_{i}$ from $v_{i}$ to $p(\eta)$-or to $p(\nu \eta)$-is given by

$$
d_{\mathrm{i}}=\sinh ^{-1}\left(\frac{\tanh r}{\tan \frac{1}{2} \alpha_{\mathrm{i}}}\right) \quad \text { ([2], p. 147, Theorem 7.11.2). }
$$

If $v$ is an ideal vertex, the angle is zero and the distance from the contact point is infinite, so the formula is still correct with the only reasonable interpretation.

We now define a set of matching isometries compatible with $\mu_{1}$. Suppose first that $v_{i}$, $v_{i+1}$ are both ordinary vertices and let $\eta=\left(v_{i}, v_{i+1}\right), \mu_{1} \eta=\left(v_{k}, v_{l}\right)$. Then $v_{i}$, $v_{k}$ belong to the same cycle so $\alpha_{i}=\alpha_{k}$ and $d_{i}=d_{k}$. Similarly $d_{i+1}=d_{l}$. The lengths of the edges $|\eta|$, $\left|\mu_{1} \eta\right|$ are equal and there is a unique isometry $T(|\eta|)$ satisfying 7.1.2 and mapping $|\eta|$ to $\left|\mu_{1} \eta\right|$. The condition $d_{i}=d_{k}$ ensures that $T(|\eta|)$ maps the contact point $p(\eta)$ to $p\left(\mu_{1} \eta\right)$.

If one of the vertices $v_{i}, v_{i+1}$ is an ideal point, $T(|\eta|)$ can be constructed uniquely so as to map the ordinary vertex on its mate, and again it will map $p(\eta)$ to $p\left(\mu_{i} \eta\right)$, because their distances from the ordinary vertices are finite and equal. If, however, $v_{i}$ and $v_{i+1}$ are both ideal vertices, there are infinitely many isometries mapping $\eta$ to $\mu_{1} \eta$ and satisfying 7.1.2. Only one of these maps $p(\eta)$ to $p\left(\mu_{1} \eta\right)$, and we define $T(|\eta|)$ to be this one.

We verify next that the polygon $\left(P, \mu_{1}, m\right)$ with the $T(|\eta|)$ as matching isometries is a geometric weighted marked polygon. The angle condition 7.1.4 is an immediate consequence of the choice of angles $\alpha_{i}$. We therefore need only verify 7.1.5.

Suppose then that $i(\eta)$ is an ideal vertex $v$, and let $l=l(\eta)$. For $r=1,2, \ldots$, define $\gamma_{r}=T(|\tau \eta|) T\left(\left|\tau^{2} \eta\right|\right) \ldots T\left(\left|\tau^{r} \eta\right|\right)$, and let $\gamma_{0}=$ id. Then we assert

$$
\gamma_{r}\left(i\left(\tau^{r} \eta\right)\right)=v .
$$

Proof by induction on $r$. Make the induction hypothesis that $\gamma_{r-1}\left(i\left(\tau^{r-1} \eta\right)\right)=v$, certainly satisfied for $r=1$.

Since $T\left(\tau^{r} \eta\right)$ maps $\tau^{r} \eta$ on $\mu \tau^{r} \eta$, it maps the initial point of the one on the other, so

$$
T\left(\tau^{r} \eta\right) i\left(\tau^{r} \eta\right)=i\left(\mu \tau^{r} \eta\right)=i\left(\nu \mu \tau^{r} \eta\right)=i\left(\tau^{i-1} \eta\right)
$$

Applying $\gamma_{r-1}$ to both sides we derive

$$
\gamma_{r} i\left(\tau^{r} \eta\right)=\gamma_{r-1} i\left(\tau^{r-1} \eta\right)
$$

and 8.4 is proved by induction.

Next we have

8.5. $\gamma_{r}\left(p\left(\tau^{r} \eta\right)\right)$ and $p(\eta)$ are on the same $v$-horocycle.

To prove 8.5 observe that $T\left(\tau^{r} \eta\right)$ maps $p\left(\tau^{r} \eta\right)$ on $p\left(\mu \tau^{r} \eta\right)=p\left(\nu \tau^{r-1} \eta\right)$. Now by 5.3 the contacts with the inscribed circle of two edges with the same initial point lie on a horocycle through that initial point; so $T\left(\tau^{r} \eta\right) p\left(\tau^{r} \eta\right)$ and $p\left(\tau^{r-1} \eta\right)$ lie on the same $i\left(\tau^{r-1} \eta\right)$-horocycle. Applying the isometry $\gamma_{r-1}$, which, by 8.4 , maps $i\left(\tau^{r-1} \eta\right)$ on $v$, we deduce that $\gamma_{r} p\left(\tau^{r} \eta\right)$ and $\gamma_{r-1}\left(p\left(\tau^{r-1} \eta\right)\right)$ lie on the same $v$-horocycle. 8.5 follows by repeated application.

Let us now put $r=l$. Since $\tau^{l} \eta=\eta, \gamma_{l}$ maps $v$ on itself by 8.4. Also $\gamma_{l}$ preserves 
orientation because it maps $P_{1}$ outside the angle between $\eta$ and $\gamma_{t} \eta$ while fixing $v$. Therefore by Lemma $5.2 \gamma_{l}$ is parabolic and 7.1.5 is proved.

By the Poincare Existence Theorem, the group $\Gamma=\left\langle T(\eta): \eta \in E\left(P_{1}\right)\right\rangle$ is discrete and has $P_{1}$ as a fundamental polygon. We complete the proof by showing that $P_{1}$ is the Dirichlet polygon for $\Gamma$ at the centre $c$ of the inscribed circle.

Let $\eta$ be any directed edge of $P_{1}$. By construction, $T(\eta) p(\eta)=p(\mu \eta)$. Applying $T(\eta)^{-1}$, we see that $T(\eta)^{-1} p(\mu \eta)=p(\eta)$ is the foot of the perpendicular from the point $T(\eta)^{-1} c$ to $\eta$. Thus $\eta$ is part of the perpendicular bisector of the segment $\left(c, T(\eta)^{-1} c\right)$ and the half-plane containing $P_{1}$ and bounded by this perpendicular bisector is

$$
H_{\eta}=\left\{z \in U: d(z, c) \leqslant d\left(z, T(\eta)^{-1} c\right)\right\} \text {. }
$$

Now $P_{1}$, being a convex polygon. is the intersection of the half-planes bounded by its sides. Thus it is defined by a finite subset of the inequalities definining the Dirichlet polygon $F(\Gamma, c)$, so that $F \subset P_{1}$. Since $F$ and $P_{1}$ are both fundamental polygons, they have the same finite area, and since both are closed and convex, they are equal.

9. Positive matchings. Suppose that we have an undirected edge $e \in E_{0}(P)$. Once we direct it by choosing one of its two endpoints as initial vertex to obtain a directed edge $\eta$, which we say is positively directed, we can direct all the edges coherently to obtain the set $E^{+}(P)=\left\{\eta, \sigma \eta, \ldots, \sigma^{n-1} \eta\right\}$ of positively directed edges. The complement $E(P) \backslash E^{+}(P)$ is denoted by $E^{-}(P)$ and we have $E^{-}(P)=\rho E^{+}(P)$. A polygon with a choice of one of the two $\langle\sigma\rangle$-orbits as the set of positively directed edges is said to be oriented.

DefintTion 9.1. The edge-matching $\mu$ is positive if $\eta \in E^{+}(P)$ implies $\mu \eta \in E^{-}(P)$.

Positive edge-matchings are precisely those associated with Fuchsian groups, and can be described combinatorially by permutations of degree $n$ instead of $2 n$. For if $\mu$ is a positive edge-matching, the permutation $\mu \rho$ maps $E^{+}(P)$ to $E^{+}(P)$. Let $\mu^{*}$ be the restriction of $\mu \rho$ to $E^{+}(P)$. Since $\mu \rho=\rho \mu, \mu$ is known when $\mu^{*}$ is known, and, if $\sigma^{*}$ is the restriction of $\sigma$ to $E^{+}(P)$, then the positive matching is known up to isomorphism when we know the pair of permutations $\left(\mu^{*}, \sigma^{*}\right)$ of degree $n$, where $\mu^{* 2}=$ id and $\sigma^{*}$ is an $n$-cycle. Two such permutation-pairs $\left(\mu_{1}^{*}, \sigma_{1}^{*}\right)\left(\mu_{2}^{*}, \sigma_{2}^{*}\right)$ define isomorphic edge-matchings if and only if there is a permuation $\lambda$ such that $\lambda \mu_{1}^{*}=\mu_{2}^{*} \lambda$ and either $\lambda \sigma_{1}^{*}=\sigma_{2}^{*} \lambda$ or $\lambda \sigma_{1}^{*}=\sigma_{2}^{*-1} \lambda$, the latter possibility occurring if the isomorphism reverses the polygon's orientation.

In this case too, the initial vertex function $i: E^{+}(P) \rightarrow V$ is a bijection, so we can regard the vertices, each identified with the positively directed edge of which it is initial point, as being permuted by $\mu^{*} \sigma^{*}$, which is the restriction $\tau^{*}$ of $(\mu \rho)(\rho \nu)=\tau$ to $E^{+}(P)$. Vertex-cycles are thus permutation-cycles in a strict sense.

10. Surface groups. Let $\Phi=\Phi_{\mathrm{g}}$ be the fundamental group of a compact orientable surface of genus $g \geqslant 2$ and let $S_{0}(\Phi)$ be the space of all Fuchsian groups isomorphic to $\Phi$, topologized as in [3], [10]. Let $p$ be a fixed base-point. Then $S_{0}(\Phi)$ is a non-compact manifold of dimension $6 \mathrm{~g}-3$ and the points $\Gamma \in S_{0}(\Phi)$ can be labelled according to the standard edge-matching of the Dirichlet polygon $F(\Gamma, p)$. This labelling will dissect the manifold 
$S_{0}(\Phi)$ into subspaces, each subspace consisting of all points with the same label. The picture obtained is essentially independent of the base-point $p$, because $G$ acts transitively on $U$ and, if $\gamma \in G$, then $F(\Gamma, \gamma p)=\gamma F\left(\gamma^{-1} \Gamma \gamma, p\right)$.

If $\Gamma \in S_{0}(\Phi)$, the standard weight of every cycle is 1 , since a weight greater than one indicates a point with a non-trivial $\Gamma$-stabilizer ([9], p. 125-6). For the same reason, the standard edge-matching $\mu$ never maps a directed edge $\eta$ on $\rho \eta$, or, what is the same thing, $\mu^{*}$ never maps $\eta$ to itself; for then the matching isometry would fix the midpoint of $|\eta|$.

DEFINTIION 10.1. A generic surface matching is a positive edge-matching such that $\mu^{*}$ is a regular permutation of order 2 and every vertex-cycle has length 3 .

It follows from [2] Theorem 9.4.5, p. 232, that, for almost all $\Gamma \in S_{0}(\Phi), F(\Gamma, p)$ will have a generic surface matching. It is therefore important to make a special study of generic surface matchings, given abstractly by the set of regular involutions $\mu^{*}$ such that $\mu^{*} \sigma^{*}$ is a regular permutation of order 3 , where $\sigma^{*}=(12 \ldots n)$. We call such an involution a cubic involution, because in this case the graph in the quotient surface given by the edges of the Dirichlet polygon is a cubic graph.

LEMMA 10.2. If a cubic involution of degree $n$ exists, then $n=6(2 g-1)$, where $g$ is a positive integer.

Proof. For regular permutations of order 2 and 3 to exist, $n$ must be divisible by 6 . Since $n$ is even, $\sigma^{*}$ is an odd permutation, while $\mu^{*} \sigma^{*}$, consisting of cycles of length 3 , is an even permutation. Hence $\mu^{*}$ is an odd permutation, so $n$ is twice an odd number.

This can also be seen geometrically, in a manner which identifies $g$ with the genus of the compact orientable surface $P / \mathfrak{q}$ of Euler characteristic $2-2 \mathrm{~g}$. Counting faces, edges and vertices on the quotient surface, we find

$$
2-2 g=1-\frac{1}{2} n+\frac{1}{3} n, \text { so } n=6(2 g-1) \text {. }
$$

There is precisely one cubic involution of degree 6 , namely $(14)(25)(36)$. Since $g=1$, this is not realized by any Fuchsian group; but it is of course realized in the Euclidean plane as the classical tiling by hexagons.

Cubic involutions were studied by Hussein [8], who obtained the following formula for the number of cubic involutions of degree $n=6(2 g-1)$ :

$$
C(g)=\frac{n !}{2^{n / 2}\left(\frac{n}{2}\right) ! 3^{n / 3}\left(\frac{n}{3}\right) !} \sum_{r=0}^{n-1}(-1)^{r} a_{r} b_{r} d_{r}^{-1},
$$

where $d_{r}$ is the bonomial coefficient $\left(\begin{array}{c}n-1 \\ r\end{array}\right)$ and $a_{r}, b_{r}$ are given by the expansions

$$
\begin{aligned}
(x+1)\left(x^{2}-1\right)^{(n / 2)-1} & =\sum_{r=0}^{n-1} a_{r} x^{n-r-1} \\
\left(x^{2}+x+1\right)\left(x^{3}-1\right)^{(n / 3)-1} & =\sum_{r=0}^{n-1} b_{r} x^{n-r-1}
\end{aligned}
$$


Hussein computed $C(\mathrm{~g})$ in his thesis up to $g=17$, and his results agree with the formula

$$
C(g)=\frac{(6 g-3) !}{g !(3 g-2) ! 2^{2 g-1} 3^{g}}
$$

which I have found empirically. At present, however, I lack a proof.

10.3 gives $C(2)=105, C(3)=50050$, and the function $C(\mathrm{~g})$ grows quite rapidly with g. Two distinct cubic involutions can give isomorphic edge-matchings if one is derived from the other by a symmetry of the polygon. For example, there are 8, not 105, distinct isomorphism classes of generic surface matchings of genus 2 . These are listed in a diagram on p. 267 of [7], which appeared in print eighty-eight years ago!

11. Generic curves and the modular group. A generic surface matching is associated with three permutations, $\mu^{*}$ of order $2, \tau^{*}$ of order 3 and $\sigma^{*}$ of order $n=6(2 g-1)$. Since $\tau^{*}=\mu^{*} \sigma^{*}$, the triple can be defined by any two of them. Now the classical modular group $\Gamma(1)$ is the free product of the cyclic groups of orders 2 and 3 generated by $T, V$ respectively, where $T z=-1 / z, V z=-1 /(z+1)$. Each generic surface matching of degree $n$ defines a permutation representation of $\Gamma(1)$ of degree $n$ by mapping $T \rightarrow \mu^{*}, V \rightarrow \tau^{*}$. Thus cubic involutions correspond to subgroups of index $n$ and level $n$ which are cycloidal and torsion-free. See for instance [13].

That gives a purely algebraic connection between cubic involutions and $\Gamma(1)$. We show in this section that there is also a close geometric link. Motivated by the construction of $\S 8$, we call a Dirichlet polygon $F(\Gamma, p)$ circular if it has an inscribed circle which touches all its edges, and then we call the group $\Gamma$ circular at $p$. Whether $F(\Gamma, p)$ is circular or not, all points of a vertex cycle are equidistanct from $p$. Therefore if $F$ is circular, all the angles at a vertex-cycle are equal. To obtain a group $\Gamma$ which is circular at $p$ we must therefore use the construction of $\$ 8$ and we deduce

THEOREM 11.1. Two groups $\Gamma_{1}, \Gamma_{2}$ which are circular at $p$ with isomorphic standard markings and weightings for their Dirichlet polygons are conjugate under a rotation or reflection fixing $p$.

Let us now consider the surface groups circular at $p$ with quotient space of genus $g$ and with generic surface matchings for their Dirichlet polygons at $p$. All cycles have length 3 and weight 1 , so the Dirichlet polygon for such a group is a regular $n$-gon, $n=6(2 g-1)$, with all its angles equal to $2 \pi / 3$. It is remarkable that we obtain the same Dirichlet polygon regardless of which generic surface matching is involved. To remove the ambiguity given by the possibility of rotations and reflections, let us fix one such regular $n$-gon with centre at $p$, orient it, and label its positively directed edges $1,2, \ldots, n$ in order, so that $\sigma^{*}=(12 \ldots n)$. Then each cubic involution of degree $n$ defines a unique positive edge-matching of $P$ and, as in $\S 8$, for each such involution $\mu$ we have a surface group $\Gamma_{\mu}$ of genus $g$ with $P$ as its Dirichlet polygon. $\dagger$ Beginning with $P$ we can fill up the plane with congruent polygons, three at each vertex, to form a honeycomb $\{n, 3\}$ in Coxeter's terminology and notation $([4],[5])$. For any one of the groups $\Gamma_{\mu}$, the

$\dagger$ We change the notation by dropping the asterisk from $\mu^{*}, \sigma^{*}$ since the original $\mu, \sigma$ will no longer be needed. 
honeycomb is its Dirichlet tessellation consisting of $P$, all its $\Gamma_{\mu}$-images and all their edges and vertices. Every transformation of $\Gamma_{\mu}$ is an automorphism of the honeycomb, that is, it maps the faces, edges and vertices among themselves, so all the $\Gamma_{\mu}$ are subgroups of the automorphism group (or complete symmetry group) of $\{n, 3\}$. This group is described in [4] p. 67, 90 (see also [5], p. 53) and is discrete. A fundamental region is given by the triangle pqr, where $r$ is the initial vertex of positively directed edge 1 and $q$ is its mid point. The automorphism group is generated by the reflections $R, S, T$ in the edges opposite $p, q, r$ respectively, with defining relators

$$
R^{2}, S^{2}, T^{2},(S T)^{n},(T R)^{2},(R S)^{3} .
$$

It is the extended triangle group $T^{*}(2,3, n)$ which contains, as a subgroup of index 2 , the Fuchsian triangle group $T(2,3, n)$ with presentation

$$
\left\langle u, v \mid u^{2}, v^{3},(u v)^{n}\right\rangle
$$

with $u=T R, v=R S([\mathbf{1 1}]$, p. 65).

Since all the $\Gamma_{\mu}$ preserve orientation, they are subgroups of the smaller Fuchsian triangle group $T(2,3, n)$. If we call the algebraic curves associated with the Riemann surfaces $U / \Gamma_{\mu}$ the central curves for the involutions $\mu$, we have proved

THEOREM 11.2. All central curves of genus $g$ are uniformized by subgroups of index $n=6(2 g-1)$ in $T(2,3, n)$.

The index is calculated from the Riemann-Hurwitz relation, or indeed more simply by counting the number of triangles in the barycentric subdivision of $P$. The central curves can also be uniformized by subgroups of the modular group. To prove this, consider the classical modular figure in the upper half-plane model $H$ of hyperbolic geometry, focussing attention on the triangle bounded by $x=-\frac{1}{2}, x=0$ and the circle $|z|=1$. We can map this triangle, by the Riemann mapping theorem, analytically on the triangle pqr, mapping $\infty$ to $p, i$ to $q$ and $-\frac{1}{2}-\frac{1}{2} i \sqrt{3}$ to $r$. This function can be extended by the reflection principle to an analytic map of $H$ into $U$ with infinite branching at the centres of all the polygons in the tessellation, and inducing the obvious homomorphism $\varphi$ of the modular group onto $T(2,3, n)$. The curve $U / \Gamma_{\mu}$, punctured at the central point $p$, is birationally equivalent to $H / \varphi^{-1}\left(\Gamma_{\mu}\right)$. The groups $\varphi^{-1}\left(\Gamma_{\mu}\right)$ are precisely the cycloidal torsion-free subgroups of $\Gamma(1)$ of index $n$ and level $n$.

It is interesting to note that the euclidean case $g=1$ also has a place here-the unique normal subgroup of index 6 and level 6 in $\Gamma(1)$ is its derived group, with quotient space a punctured equianharmonic torus associated with the tiling of the plane by regular hexagons-the original honeycomb found in nature!

\section{REFERENCES}

1. A. F. Beardon, Hyperbolic polygons and Fuchsian groups, J. London Math. Soc., 20 (1979), 247-254.

2. A. F. Beardon, The Geometry of Discrete Groups (Springer, 1983). 

257-300.

3. L. Bers, Uniformization, moduli, and Kleinian Groups, Bull. London Math. Soc. 4 (1972)

4. H. S. M. Coxeter, Regular Polytopes (London, 1948).

5. H. S. M. Coxeter and W. O. J. Moser, Generators and Relations for Discrete Groups (Springer 1957).

6. G. de Rham, Sur les polygones générateurs des groupes Fuchsiens. l'Enseignement Math 17 (1971), 49-62.

7. R. Fricke und F. Klein, Vorlesungen über die Theorie der automorphen Funktionen (Teubner, 1897).

8. A. A. Hussein Omar, On some permutation representations of $(2,3, n)$-groups $\mathrm{Ph} . \mathrm{D}$. thesis, (Birmingham, England, 1979).

9. J. Lehner, Discontinuous groups and automorphic functions Amer. Math. Soc., 1964).

10. A. M. Macbeth and D. Singerman, Spaces of subgroups and Teichmüller Space, Proc. London Math. Soc. (3) 31 (1975) 211-256.

11. W. Magnus, Non-euclidean tesselations and their Groups. (Academic Press, 1974).

12. B. Maskit, On Poincaré's theorem for fundamental polygons, Advances in Math. 7 (1971) 219-230.

13. M. H. Millington, Cycloidal subgroups of the modular group, Proc. London Math. Soc. (3) 19 (1969) 164-176.

14. H. Poincaré, Oeuvres completes. t. II.

15. C. L. Siegel, Some Remarks on Discontinuous Groups. Annals of Math. 46 (1945) 708-718.

Department of Mathematics and Statistics

UNIVERSTTY OF PITTSBURGH

PItTSBurgh PA. 15260 